\title{
Wetting Effects at a Grain Boundary
}

\author{
D. B. Abraham, ${ }^{1}$ Ville Mustonen, ${ }^{1,2}$ and A. J. Wood ${ }^{1}$ \\ ${ }^{1}$ Theoretical Physics, University of Oxford, Oxford OX1 3NP, United Kingdom \\ ${ }^{2}$ Laboratory of Computational Engineering, Helsinki University of Technology, P.O. Box 9203, FIN-02015, Finland
}

(Received 10 February 2004; published 10 August 2004)

\begin{abstract}
We consider a tier of weakened bonds along the center line of a two-dimensional Ising ferromagnet strip as a model of a grain boundary. When an interface traverses such a strip at an angle, whether or not there is a continuous pinning-depinning transition at subcritical temperature depends on this angle and the degree of bond weakening. We also study the relaxation of such a system to its equilibrium state using continuous time Monte Carlo simulation with Kawasaki dynamics; this reveals a matter transport mechanism confined to the grain boundary.
\end{abstract}

DOI: 10.1103/PhysRevLett.93.076101

PACS numbers: 68.08.Bc, 05.70.Np, 68.35.Rh

Consider a planar Ising ferromagnet with nearest neighbor interactions and a zero bulk magnetic field with a strip geometry and at a temperature $T$ below the bulk critical value $T_{c}$. By fixing the spin values to be all +1 on one edge and -1 on the other, configurations with an odd number of domain walls running on average parallel to the strip axis, which we take to be $(1,0)$, are induced; this number is effectively one as the strip length goes to infinity, because the surface tension is strictly positive. Thus, as the width of the strip diverges, we have two oppositely magnetized bulk phases separated by an interface with average orientation $(1,0)$.

Suppose the bonds in the $(0,1)$ direction between a single pair of adjacent rows of spins are weakened. Such an arrangement is a simple model of a grain boundary and forms an energetic trap for thermally fluctuating domain walls, thus bringing in the usual energy-entropy paradigm for phase transitions.

Two problems in this class have exact equilibrium statistical-mechanical solutions: when the line of defect bonds is in the middle of a strip of divergent width, the domain wall is bound for all $T<T_{c}$ [1]. If the line of defect bonds is at the edge of the lattice, there is a continuous phase transition at a temperature $T_{w}<T_{c}$, depending on the degree of bond reduction [2]; which is related to the wetting phenomenon as exemplified by sessile drops [3].

This rather curious geometric dependence has been clarified by mesoscopic modeling, depending on the idea of coarse graining to the scale of the bulk correlation length; this eliminates the internal structure of the equilibrium phases, leaving just the location of the phase separating lines which replace the interface. Clearly, such a procedure is not unique, but an exactly derived example is known [4]. The random walk analysis of such a setup by Fisher [5] accentuates the geometric subtleties of returns to the defect line, which are treated as recurrent events. The fact that these returns occur from both sides of the grain boundary in Fig. 1 increases the entropy of configurations which are bound to the interface, com- pared with one-sided returns which obtain when the grain boundary remains at, or indeed near, the edge. This increase is sufficient to differentiate sharply between these cases, in the way described in the previous paragraph. The nature of the arguments implies strongly that the specific results from the planar Ising model are in fact general.

In this Letter, we consider an interface which crosses the grain boundary at an angle implemented as in Fig. 1 by fixing the spins at the edges. When $b=1$ (no grain boundary) the optimal, most likely, path is the shortest,

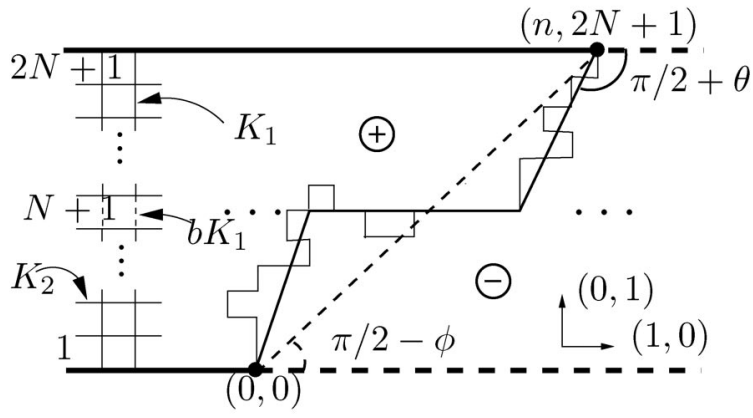

FIG. 1. A schematic picture of the interface induced by the boundary conditions described in the text. The spins at the solid horizontal boundaries are fixed to be positive and the ones at the broken horizontal boundaries are fixed to be negative. The dashed line depicts a mean macroscopic interface crossing the system at an angle $\phi$, the heavy solid line shows a typical zigzag interface configuration, where the middle section is pinned to the grain boundary. The transition studied, the GZZ transition, is the. crossover between these two configurations. The thin solid line shows a typical microscopic zigzag configuration. A perfect zigzag is formed when $\theta=0$, this corresponds to the zero temperature configuration for a system with a grain boundary $(b<1)$ and is also one of the degenerate ground states for a system without a grain boundary $(b=1)$. The bond strengths of the model are included for reference, where $K_{j}=J_{j} / k_{B} T$. The circled plus (minus) sign depicts that the bulk is on average at plus (minus) magnetization on the left (right) hand side of the interface separating the phases. 
geodesic one. But suppose $0<b<1$ (ferromagnetic grain boundary); a zigzag path as in Fig. 1 allows the interface to profit energetically from intersecting the weakened bonds, with entropic implications; a calculation is needed to see whether there is indeed a phase transition as $N \rightarrow \infty$ from the geodesic to the zigzag configuration. A simple variational calculation [6], which is analogous to deriving the modified Young formula for wetting [3], suggests that when a segment of the interface is pinned at the grain boundary, it exits from it at a definite angle, depending on $T$ and $b$, but not on $\phi$. Thus, if $\phi$ is too small, the zigzag cannot be accommodated with a positive intersection at the grain boundary; this mechanism will suppress the phase transition. Thus, another way of expressing the transition is that there is a critical angle $\phi_{c}=\theta_{c}(b, K)$; this is reminiscent of filling [7]. These intuitive ideas for a geodesic-to-zigzag (GZZ) transition are confirmed by the calculation reported here.

Finally, the striking and unanticipated precursor film phenomenon [8] in the dynamics of complete wetting from an initially sessile drop has received much recent attention [9]. There is an analogy here, in that when the zigzag configuration obtains at equilibrium, the dynamics of approach to equilibrium favors, in the main, motion of matter (in the lattice gas interpretation) along the line of defect bonds. This, as a generalization of what happens at equilibrium, may be termed dynamical confinement.

Our microscopic system is constructed by considering fixed spin or Dobrushin, boundary conditions which induce an interface to cross an Ising ferromagnetic strip of width $2 N+1$ on a quadratic lattice, as shown in Fig. 1 for orientation of axes. Coexistence of phases (as $N \rightarrow \infty$ ) is assured by imposing zero bulk field and a subcritical temperature. We model a grain boundary in such a system by weakening a horizontal line, symmetrically placed, of vertical bonds. Both the incremental free energy and the spatial dependence of magnetization will be derived. Translational invariance, essential in our formulation, is achieved by imposing cyclic boundary conditions, but then it is clear that there has to be a further spin flip on each edge. This gives a cylindrical lattice of circumference $M$, height $(2 N+1)$ with domain walls intersecting the edges at $(0,0),(k, 0),(n, 2 N+1)$, and $(n+k, 2 N+1)$. We take $M \rightarrow \infty$, followed by $k \rightarrow \infty$ and then extract the incremental free energy $F^{x}$ of a single domain wall connecting $(0,0)$ to $(n, 2 N+1)$. The additional difficulty in the calculation, one in mathematical physics, which has to be overcome is to insert the line of defect bonds. Here we give the results and focus on their physical interpretation. By calculating the ratio of the partition function for the defect strip with a domain wall to one without such a wall, we get

$$
e^{-F^{x}(N, n)}=\frac{1}{2 \pi} \int_{-\pi}^{\pi} \frac{e^{-2 N \gamma(\omega)} e^{i n \omega}}{A_{N}(\omega)} d \omega .
$$

The Onsager function $\gamma(\omega)$ is given by [10] 076101-2

$$
\cosh \gamma(\omega)=c_{1}^{*} c_{2}-s_{1}^{*} s_{2} \cos \omega,
$$

with $\exp 2 K_{j}^{*}=\operatorname{coth} K_{j}, c_{j}^{*}=\cosh 2 K_{j}^{*}, c_{j}=\cosh 2 K_{j}, s_{j}^{*}=$ $\sinh 2 K_{j}^{*}, s_{j}=\sinh 2 K_{j} \quad$ (subcriticality is determined by $\left.K_{1}^{*}<K_{2}\right)$. We have obtained $A_{N}(\omega)$ for all $N$, but here we are interested in the limiting behavior as $N \rightarrow \infty, n \rightarrow$ $\infty$, with $n=[2 N \tan \phi]$ (nearest smaller integer, say). We calculate the free energy per unit length as

$$
f^{x}(\phi, b)=\lim _{N \rightarrow \infty} F^{x}(N,[2 N \tan \phi]) / 2 N \sec \phi,
$$

by proving that in (1) $A_{N}(\omega)$ can be replaced by $A_{\infty}(\omega)$, given in a factorized form as

$$
A_{\infty}(\omega)=\frac{e^{-2 K_{2}} s_{1}\left(1+\cos \delta^{*}\right)\left(e^{\gamma(\omega)}-\lambda_{-}\right)\left(e^{\gamma(\omega)}-\lambda_{+}\right)}{4 \sinh \left(2 b K_{1}\right) \sinh \gamma(\omega)},
$$

where

$$
\lambda_{ \pm}=w_{g} \pm \sqrt{w_{g}^{2}+1-2 w_{g} c_{1}^{*} / c_{2}}
$$

with

$$
w_{g}=c_{2}\left[c_{1}^{*}-s_{1}^{*} \cosh \left(2 b K_{1}\right)\right] .
$$

Finally, the angle $\delta^{*}(\omega)$ in (4) is given by the hyperbolic triangle formula

$$
s_{1}^{*} \sinh \gamma \cos \delta^{*}=c_{2} \cosh \gamma-c_{1}^{*} .
$$

We can glean much information about (1)-(7) by considering some special cases: first, suppose $b=1$ so there is no pinning. Then $\lambda_{ \pm}= \pm 1$ and $A_{\infty}(\omega) \rightarrow e^{\gamma(\omega)} e^{-2 K_{2}}[1+$ $\left.\cos \delta^{*}(\omega)\right] / 2$ : (1) and (3) give the surface tension of an inclined interface. The integrand in (1) has a saddle point at $\omega_{s}$ where $\gamma^{(1)}\left(\omega_{s}\right)=i \tan \phi$, implying $\omega_{s}=i v_{s}$, with $v_{s}$ real. Considerable algebra makes this more explicit:

$$
\cosh \gamma\left[i v_{s}(\phi)\right]=\left(c_{1}^{*} c_{2}-\mathcal{B}^{1 / 2}\right) \cos ^{2} \phi / \cos 2 \phi,
$$

where

$$
\mathcal{B}=\frac{\cos ^{2} 2 \phi}{\cos ^{4} \phi}-\left[\left(c_{1}^{*}\right)^{2}+\left(c_{2}\right)^{2}\right] \frac{\cos 2 \phi}{\cos ^{2} \phi}+\left(c_{1}^{*} c_{2}\right)^{2} .
$$

This shows that $0 \leq v_{s}(\phi)<2\left(K_{1}-K_{2}^{*}\right)$ for $0 \leq$ $\phi<\pi / 2$, implying that to go from the contour $(-\pi, \pi)$ in (1) to the steepest descent path, no singularities are crossed; there are "bulk" branch points at $\omega= \pm 2 i\left(K_{1}-\right.$ $\left.K_{2}^{*}\right), \omega= \pm 2 i\left(K_{1}+K_{2}^{*}\right)$. As $\phi$ increases from 0 to $\pi / 2$, $v_{s}(\phi)$ increases from 0 to $2\left(K_{1}-K_{2}^{*}\right)$.

A second case of interest is $n \rightarrow \infty$, followed by $N \rightarrow$ $\infty$. There is no saddle point and (1) is controlled by the nearest pole, given by $\exp \gamma(\omega)=\lambda_{+}$, for which $\omega=$ $i v_{+}$. The incremental energy per unit length in the $(1,0)$ direction is $v_{+}(b)$ which equals $f^{x}(\pi / 2, b)$, as anticipated; holding $K_{1}, K_{2}$ constant, $v_{+}(b)$ increases monotonically with $b$ on $[0,1]$ with $v_{+}(0)=0$ and $v_{+}(1)=2\left(K_{1}-K_{2}^{*}\right)$. Thus $v_{+}(b)<\tau(1,0)$ where $\tau(1,0)$ is the surface tension for a free interface with average

076101-2 
direction $(1,0)$. Only for $b=1$ do we have $v_{+}(1)=$ $\tau(1,0)$. It is tempting, and indeed correct, to assume that the interface is bound to the defect line, in accordance with Fisher's criterion [5].

The new factor in the current problem is that for $0<$ $\phi<\pi / 2$, the simple pole of $A_{\infty}(\omega)$ in (4) at $\omega=i v_{+}(b)$ can cross the steepest descent path. The intersection is at

$$
w_{g}^{2}\left(\tan ^{2} \phi-1\right)+c_{2}\left(2 w_{g} c_{1}^{*}-c_{2}\right)=0,
$$

which defines the phase boundary for the "GZZ" transition. Inserting (6) into (10) shows that this condition is independent of $K_{2}$, which enters the discussion since we require $K_{1}^{*}<K_{2}$ as well as (10). The special case $\phi=$ $\pi / 4, K_{1}=K_{2}=K$ reduces to solving $\exp 2 b K=\cosh 2 K$ with $\sinh 2 K>1$; in this case, there is a $b_{c}$ such that, for $0<b<b_{c}$, there is no solution for $K$ with $\sinh 2 K>1$. Clearly $v_{s}$ is independent of $b$, so for small enough $b$, the asymptotics of (1) is pole dominated. The asymptotics of (3) is $f^{x}(\phi, b)=\gamma(i v) \cos \phi+v \sin \phi$ with $v=v_{+}(b)$ for pole domination, but $v=v_{s}(\phi)$ from (8) with saddle point domination. For fixed $\phi$ the boundary has been depicted for three cases in Fig. 2 with the corresponding Monte Carlo (MC) simulation results.

A geometrical interpretation of this phase transition can be obtained from the magnetization $m(j, n)=$ $\langle\sigma(j, N-n)\rangle$, derived as a generalization of the original interface profile calculation [12] to include the grain boundary. When the parameters are such that the thermodynamics in the preceding paragraph is described by the saddle point, the inclined interface result is recaptured [11]; thus the grain boundary is ignored. In the pole dominated case, after quite some analysis, the result is

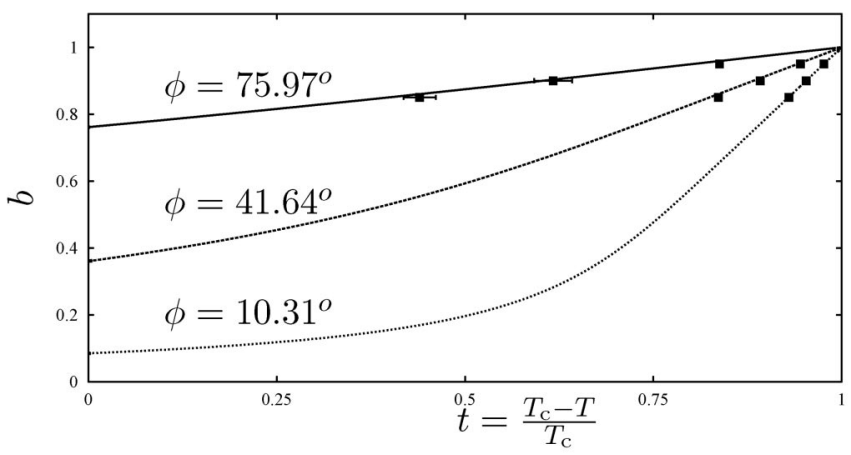

FIG. 2. Lines depict the phase diagram of the "GZZ" transition for three different angles calculated from the exact result (10). Phase transition points defined by MC simulation for these angles are plotted for $b=0.95,0.9,0.85$. We used $N$-fold implementation of the Wang-Landau sampling [16] and defined the transition point from susceptibility peaks. System sizes used were $63 \times 14\left(\phi=75.97^{\circ}\right), 47 \times 46\left(\phi=41.64^{\circ}\right)$, and $23 \times 78\left(\phi=10.31^{\circ}\right)$. The error bars are the standard deviations over ten realizations. Finite size effects change the phase diagram obtained from MC simulations showing that the transition temperature is lowered; see, for example, [17].

$$
\begin{aligned}
m(j, n)= & m^{*}-\frac{m^{*} e^{(N-n) \gamma\left(i v_{+}\right)+j v_{+}}}{2 \pi} \\
& \times \int_{-\pi}^{\pi} \frac{e^{-i j \omega-(N-n) \gamma(\omega)} f(\omega) d \omega}{e^{i \omega} e^{-v_{+}}-1} .
\end{aligned}
$$

The function $f(\omega)$ will not be specified, since it will only be needed at the saddle point in the steepest decent analysis of (11). This saddle point is given by $\omega=-i v_{s}$ with $\gamma^{(1)}\left(i v_{s}\right)=j /(N-n)$. The leading behavior in (11) is got by linear approximation to the denominator and usual quadratic one in the $\gamma(\omega)$ expansion, with the result that the integral is approximated by

$$
e^{(N-n)\left[\gamma\left(i v_{+}\right)-\gamma\left(i v_{s}\right)\right]} f\left(i v_{s}\right) \frac{e^{j\left(v_{+}-v_{s}\right)}}{2 \pi} \int_{-\infty}^{\infty} \frac{e^{-x^{2}} d x}{u+i x},
$$

where

$$
u=\left[1-e^{-\left(v_{s}-v_{+}\right)}\right]\left[\frac{(N-n) \gamma^{(2)}\left(i v_{s}\right)}{2}\right]^{1 / 2} .
$$

Recall that $v_{s}$ here is determined by $j$ and $(N-n)$ and that (12) is related to the error function [13]. The most interesting case is when $v_{s}-v_{+}=O(1 / \sqrt{N-n})$; this implies that

$$
m(j, n) \sim \frac{2 m^{*}}{\sqrt{\pi}} \int_{0}^{u} e^{-x^{2}} d x,
$$

where

$$
u \sim\left(j-j_{0}\right) /\left[2(N-n) \gamma^{(2)}\left(i v_{+}\right)\right]^{1 / 2},
$$

with $\gamma^{(1)}\left(i v_{+}\right)=j_{0} /(N-n)$. Thus we have Gaussian fluctuations of the interface beginning at the origin about the mean angle $\theta$ (see Fig. 1) with $\tan \theta=j_{0} /(N-n)$ which is thus given in terms of $b$ and the $K_{j}$ but which is independent of $\phi$. The $\gamma^{(2)}\left(i v_{+}\right)$term in (15) brings in the surface stiffness as a control parameter for the capillary fluctuations of the interface [14].

As mentioned above, Fig. 2 displays some fits to our exact equilibrium results of MC simulations; these demonstrate that finite size effects have been handled properly and are used as a reference to ensure that our simulations with the continuous time MC algorithm and Kawasaki dynamics [15] approach the correct equilibrium. A very interesting question is how the matter is transported in thermalizing from the $T=0$ configuration as an initial configuration (interface in a zigzag configuration with $\theta=0$ in Fig. 1) to equilibrium at $T>0$. The standard chemical physical idea is that the most important paths are those involving least excitation energy. What we show here is that matter flows along the grain boundary, by creating spin flip pairs aligned on $(0,1)$, which dissociate and then diffuse freely along either side of the boundary, except for occasional trapping or collision. They are then absorbed in the corners formed by the junctions of the vertical interface sections and the interface section 
pinned at the grain boundary, promoting relaxation to the equilibrium wedge angle.

We considered two systems, one with $(b=0.85)$ and one without $(b=1)$ a grain boundary. As depicted in Fig. 1, we fix spins on the upper and lower boundaries to force an interface in the system; furthermore an antiperiodic boundary condition is used in the $(1,0)$ direction to remove the extra interface. In order for the system to reach equilibrium, matter must to be transported through the system. The main interest of the matter transfer mechanism is the movement in the $(1,0)$ direction. Accordingly, we define the total mass transfer for each row of spins as

$$
S_{j}^{b}\left(t_{m c}\right)=S_{j}^{b}\left(t_{m c}-1\right)+\sum_{i} f\left(\sigma_{i, j}, \sigma_{i+1, j}\right) \sigma_{i+1, j},
$$

where $f=1$ if the spin pair $\sigma_{i, j}, \sigma_{i+1, j}$ is flipped, otherwise $f=0, t_{m c}$ is the number of MC steps, and $b$ the bond weakening in the middle. We studied a system with $M=$ $139,2 N+2=34, \phi=75.97^{\circ}$, and $t=T_{c}-T / T_{\mathrm{c}}=0.65$. The system with the grain boundary will relax to a zigzag configuration since the field $b<b_{c}=0.91$ [calculated from (10)] for the given parameters.

After the simulation has equilibriated, we calculate the normalized average total mass transfer $I_{j}^{b}=$ $\left\langle S_{j}^{b}\right\rangle / \sum_{j}\left\langle S_{j}^{b}\right\rangle$ formed over $450 \mathrm{MC}$ realizations. Looking at these fractions for the $b=1$ case, we find that the middle two rows contribute less than quarter of the total, i.e., $I_{17}^{1}+I_{18}^{1}=0.23$, the next ones $I_{16}^{1}+I_{19}^{1}=0.14$, $I_{15}^{1}+I_{20}^{1}=0.09, I_{14}^{1}+I_{21}^{1}=0.06$, and all the rest $\sim 0.02$ each (this is diffusion through the bulk phase). Consider now the system with a grain boundary $(b=$ 0.85 ). The two middle rows, i.e., the grain boundary, contribute more than two thirds of the total, $I_{17}^{0.85}+$ $I_{18}^{0.85}=0.68$ and rest of the rows each contribute $\sim 0.02$. Comparing these fractions leads to the following conclusions. While the system without a grain boundary equilibrates using capillary fluctuations, the case with the grain boundary will relax using an alternative, and novel, mechanism; the section of the interface pinned to the grain boundary will act as a diffusive guide for the particles. It is also important to notice that in the case $b=1$ the amount of total mass needed to transfer before the system reaches equilibrium is greater $\sum_{j}\left\langle S_{j}^{1}\right\rangle / \sum_{j}\left\langle S_{j}^{0.85}\right\rangle=1.47$, but still $\left(\left\langle S_{17}^{1}\right\rangle+\left\langle S_{18}^{1}\right\rangle\right) /$ $\left(\left\langle S_{17}^{0.85}\right\rangle+\left\langle S_{18}^{0.85}\right\rangle\right)=0.51$, meaning that the absolute number of particles using the middle two rows is doubled when the grain boundary is introduced. This means that by introducing a defect line in the system one spatially confines the bulk of the mass transport to the minimum energy pathway.

In summary, we have shown that, with inclined crossing of the grain boundary, depending on the degree of bond deficiency and angle, there are three possible scenarios. First, the interface can stay pinned for all $T<T_{c}$; second, there is a continuous pinning-depinning transition as described in this Letter; and third, there is no pinning at all. This is the extension of the Fisher scenario for the interior grain boundary [5]. Finally, our matter conserving $\mathrm{MC}$ analysis of the transport of matter in this system indicates that most of this takes place along the grain boundary in relaxation processes, prompting us to term this dynamical confinement.

D. B. A. and A. J.W. acknowledge financial support from the EPSRC under Grants No. GR/M04426 and No. GR/R83712/01, respectively. A. J.W. would also like to thank the Royal Commission for the Exhibition of 1851. V. M. was partially supported by the Academy of Finland, Research Centre for Computational Science and Engineering, Project No. 44897 (Finnish Centre of Excellence Programme 2000-2005). Computing time made available by collaboration of Laboratory of Computational Engineering (Helsinki University of Technology) and Wolfson College (Oxford) is greatly appreciated.

[1] D. B. Abraham, J. Phys. A 14, L369 (1981).

[2] D. B. Abraham, Phys. Rev. Lett. 44, 1165 (1980).

[3] D. B. Abraham and L-F. Ko, Phys. Rev. Lett. 63, 275 (1989).

[4] D. B. Abraham and F. T. Latremoliére, Phys. Rev. Lett. 77, 171 (1996).

[5] M. E. Fisher, J. Stat. Phys. 34, 667 (1984).

[6] D. B. Abraham, A. O. Parry, and A. J. Wood, Europhys. Lett. 60, 106 (2002).

[7] A. O. Parry, C. Rascón, and A. J. Wood, Phys. Rev. Lett. 83, 5535 (1999); A. O. Parry, A. J. Wood, E. Carlon, and A. Drzewiński, Phys. Rev. Lett. 87, 196103 (2001); D. B. Abraham and A. Maciolek, Phys. Rev. Lett. 89, 286101 (2002).

[8] S. F. Burlatsky, G. Onshanin, A. M. Cazabat, M. Moreau, and W. P. Reinhard, Phys. Rev. E 54, 3832 (1996).

[9] D. B. Abraham, R. Cuerno, and E. Moro, Phys. Rev. Lett. 88, 206101 (2002).

[10] L. Onsager, Phys. Rev. 65, 117 (1944).

[11] D. B. Abraham and P. J. Upton, Phys. Rev. B 37, 3835 (1988).

[12] D. B. Abraham and P. Reed, Phys. Rev. Lett. 33, 377 (1974); Commun. Math. Phys. 49, 35 (1976).

[13] M. Abramovitz and I. A. Stegun, Handbook of Mathematical Functions (Dover, 1972), p. 297, Eqs. 7.1.3, 7.1.4.

[14] M. P. A. Fisher, D. S. Fisher, and J. D. Weeks, Phys. Rev. Lett. 48, 368 (1982).

[15] M. E. J. Newman and G. T. Barkema, Monte Carlo Methods in Statistical Physics (Oxford University Press, New York, 1999).

[16] F. Wang and D. P. Landau, Phys. Rev. Lett. 86, 2050 (2001); B. J. Schulz, K. Binder, and M. Müller, Int. J. Mod. Phys. C 13, 477 (2002).

[17] D. B. Abraham, V. Mustonen, and A. J. Wood, Europhys. Lett. 63, 408 (2003). 